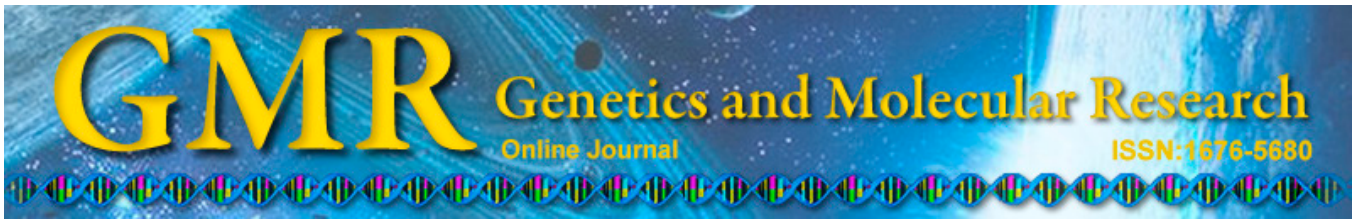

\title{
Study of the relationship between the expression of nerve growth factor and aneurysm formation and prognosis
}

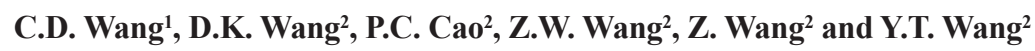 \\ ${ }^{1}$ Centre Laboratory, Weifang People's Hospital, Shandong, China \\ ${ }^{2}$ Department of Neurosurgery, Weifang People's Hospital, Shandong, China \\ Corresponding author: Y.T. Wang \\ E-mail: yutingwang929@163.com
}

Genet. Mol. Res. 14 (2): 4269-4275 (2015)

Received June 16, 2014

Accepted September 23, 2014

Published April 28, 2015

DOI http://dx.doi.org/10.4238/2015.April.28.8

\begin{abstract}
We sought to investigate the effect of nerve growth factor (NGF) expression on the formation and prognosis of cerebral aneurysms. Forty-eight cases were selected following a diagnosis of cerebral aneurysm using computed tomography angiography and surgical confirmation. Thirty-four cases of healthy deaths were also chosen. The tissue was tested for NGF expression changes by reverse-transcription PCR, Western blot and histopathology, and NGF expression was compared between the cerebral aneurysm and healthy groups. The expression level of NGF in cerebral aneurysm tissue was significantly increased over that observed in control tissue. The abnormal expression of NGF is related to cerebral aneurysms. The elevated expression of NGF in cerebral aneurysms may be associated with a poor prognosis.
\end{abstract}

Key words: Nerve growth factor; Brain aneurysm; CTA; RT-PCR 


\section{INTRODUCTION}

Cerebral aneurysm is a type of tumor in the cerebral artery branches. This disease has high mortality and recurrence rates. It is the primary cause of subarachnoid hemorrhage, which is a serious condition that often follows cerebral thrombosis and hemorrhage. Based on their location, cerebral aneurysms can be classified as internal carotid artery aneurysms, anterior cerebral artery aneurysms, and basilar artery aneurysms (Schwenk et al., 2010; Michel et al., 2011). Previous studies found that internal carotid artery bifurcation occurs in approximately $40 \%$ of all intracranial aneurysms, in which the posterior traffic artery branches are most frequent. Aneurysms of the anterior cerebral artery, including branch aneurysms of the anterior communicating artery, account for $30 \%$ of the cases of cerebral aneurysms. Among them, anterior cerebral artery aneurysms of the end position account for $5 \%$ of cases, and middle cerebral artery aneurysms account for $20 \%$, which primarily occur in the middle cerebral and trigeminal arteries; other locations account for $5 \%$ of cases. Multiple aneurysms can occur in the brain arteries, including the internal carotid posterior communicating artery and the ophthalmic artery branch (Martinez-Pinna et al., 2010; Golledge et al., 2010).

Nerve growth factor (NGF) is a neurotrophic factor that combines with the target cell's surface specific nerve growth factor receptors (van Kuijk et al., 2010). Previous studies have pointed out that NGF expression is elevated in many kinds of tumors. The reason may be that the NGF not only promotes cell growth, but it can also bind to the high-affinity receptor on the perineurium. This binding can improve the micro-environment to facilitate cell growth and to promote tumor cell migration (Ramos-Mozo et al., 2012); this mechanism has been confirmed in bladder cancer, breast cancer, and stem cell carcinoma (Seidel et al., 2010; O'Hare et al., 2010; Chan et al., 2013). However, the relationship between NGF expression and cerebral aneurysms has not been reported. The aim of this study was to evaluate the expression of NGF in patients with cerebral aneurysms in our hospital and to determine whether a relationship between NGF and cerebral aneurysms exists, providing data to support further research on NGF.

\section{MATERIAL AND METHODS}

\section{General data}

Forty-eight patients who were diagnosed as having cerebral aneurysms at Shandong Weifang People's Hospital were selected for this study. The study population consisted of 18 men and 30 women aged 48-72 years, with an average age of 55 years. Thirty-four healthy controls were also selected, including 17 men and 17 women). In this group, there was no disease of the brain; the brain tissue structure was normal and no cerebral aneurysms were observed. Postoperatively, tumor tissue was frozen immediately for testing in the laboratory. RNA was extracted from $1 \mathrm{~g}$ tissue and stored at $-80^{\circ} \mathrm{C}$ until analysis. Reverse transcription-polymerase chain reaction (RT-PCR) was used to detect RNA expression. Western blot analysis was also performed using another $1 \mathrm{~g}$ tissue. The analysis of histopathological findings was performed by the Shandong Department of Pathology Weifang People's Hospital. The protocol for this study was approved by the review board of Shandong Weifang People's Hospital (Shandong, China), and informed consent was obtained from all subjects. 


\section{Reagents and equipment}

Standard RNA extraction and RT-PCR detection kits from Takara, Japan, were used, and tissue lysis buffer was purchased from Zitian Biotech, China. The antibody used for the Western blot experiment was from Santa Cruz Biotechnology, USA. The automatic western detector was from Beckman Coulter Inc., USA.

\section{METHODS}

\section{Computed tomography angiography detection}

Forty-eight patients underwent computed tomography angiography (CTA) performed by radiologists to determine the site of aneurysm occurrence; the results of this test formed the basis for further surgical resection.

\section{RT-PCR and Western blot detection of NGF expression}

One gram of tissue was used to extract RNA to perform RT-PCR. At the same time, Western blot analysis was carried out using tissue lysates from $1 \mathrm{~g}$ of each tissue sample. RNA extraction and RT-PCR were performed by standard protocols. For Western blot analysis, after electrophoretic separation, proteins were transferred to a nitrocellulose membrane $(18 \mathrm{~V}, 30$ $\mathrm{min})$. The membrane was placed in blocking solution at $4{ }^{\circ} \mathrm{C}$ overnight. The following day, it was placed in another blocking solution containing primary antibody at $37^{\circ} \mathrm{C}$, for $2 \mathrm{~h}$ and rinsed five times for $5 \mathrm{~min}$ in phosphate-buffered saline with Tween (PBST) prior to the addition of the secondary antibody at $37^{\circ} \mathrm{C}$ for $1 \mathrm{~h}$ and automatic detection.

\section{Production and histopathology results}

Immunohistochemical analysis was used to detect the pathological expression of NGF, which, along with tissue sectioning, was conducted using standard protocols in the Department of Pathology, followed by counter-staining with hematoxylin for 5 min, dehydration, transparent closing, and observation with a microscope. The positive NGF cells had yellow granular cytoplasm. NGF expression was evaluated as follows: strong positive, positive cells $>50 \%$; weak positive, positive cells $15-50 \%$; negative, no positive cells.

\section{Statistical analysis}

Data are reported as means \pm standard deviation. The groups were compared using the independent sample $t$-test; the SPSS version 11.0 software was used to process the data, and $\mathrm{P}$ $<0.05$ was considered to be statistically significant.

\section{RESULTS}

\section{CTA test results}

All patients underwent CTA analysis. Using this method, we could clearly observe the 
site and size of the tumors (Figure 1), indicating that CTA is advantageous for the detection of cerebral aneurysms.
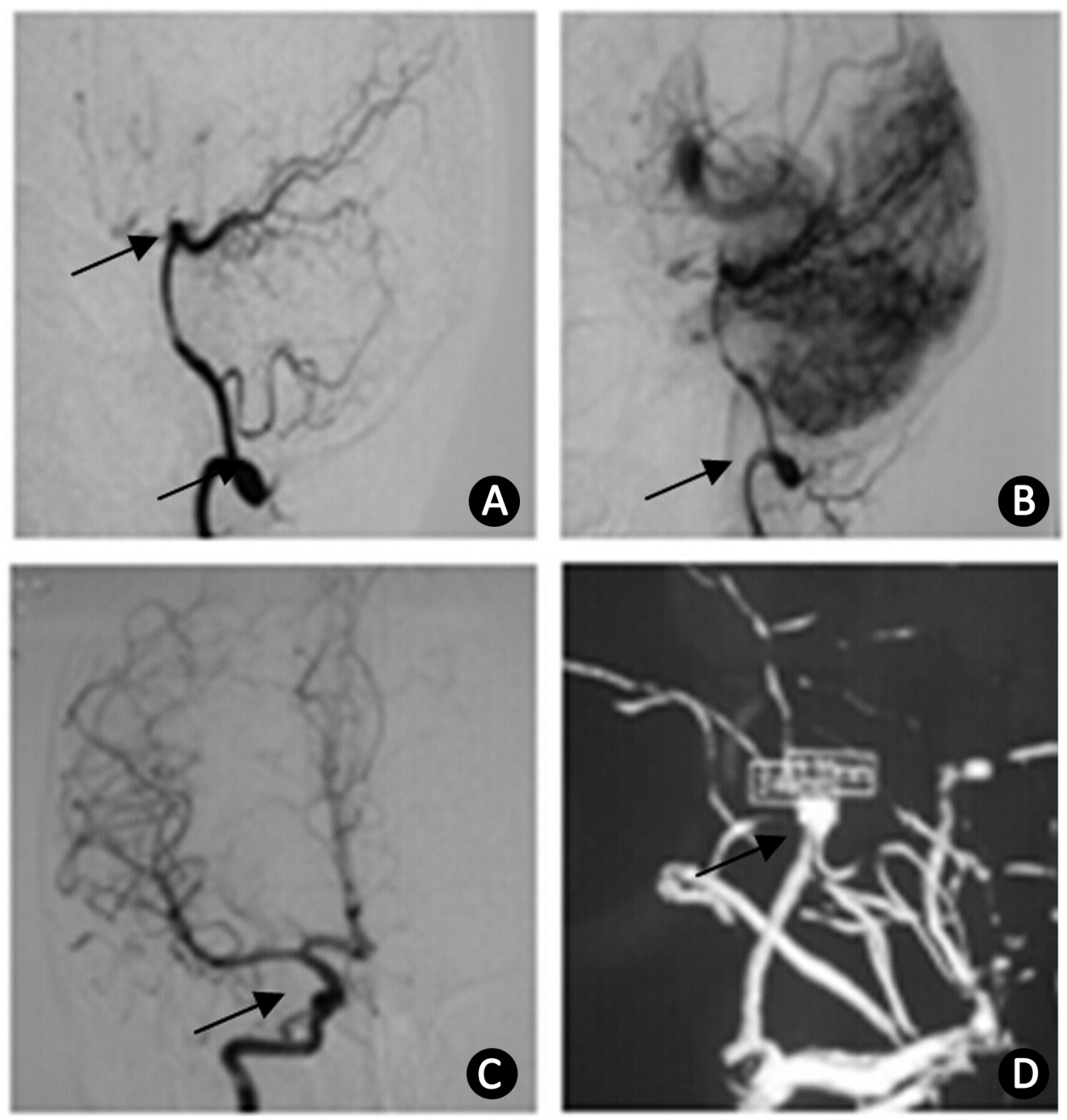

Figure 1. Aneurysm imaging. A. B. C. D. All the aneurysms are in the CTA angiography.

\section{RT-PCR and Western blot detection of NGF expression}

RT-PCR was used to detect the expression of NGF (Table 1), and the expression levels of NGF in all patients were compared with those in the healthy control group. In the patient group, the expression of NGF was significantly increased compared to the control group $(\mathrm{P}<$ 0.05 ), while no difference was observed between the two groups of patients using Western blot analysis. The RT-PCR detection results are shown in Figure 2. 
Table 1. Nerve growth factor (NGF) RT-PCR test results.

\begin{tabular}{lcc}
\hline Group & Cases (N) & NGF RNA expression \\
\hline Patients & 48 & $4.40 \pm 0.36$ \\
Controls & 34 & $1.01 \pm 0.24$ \\
$t$-value & 2.356 & 4.678 \\
P value & 0.062 & $0.036^{*}$ \\
\hline
\end{tabular}

*Compared with the healthy control group, $\mathrm{P}<0.05$.

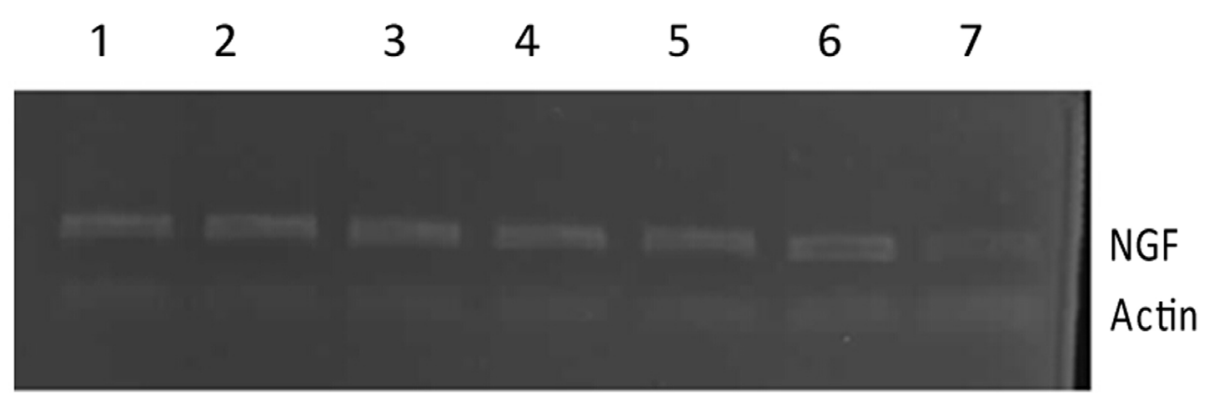

Figure 2. Western blot test NGF expression. Lanes 1-6 = NGF expression of patients with cerebral artery. Lane $7=$ NGF expression of the health population.

\section{Analysis of biopsy cases}

Histological analysis showed that NGF was mainly located in the cytoplasm, with brown granular or diffuse distribution. The expression of NGF in patients with cerebral aneurysms was higher than that in the control group There was a significant difference between the two groups using analysis with NGF integrated optical density $(\mathrm{P}<0.01$; Table 2$)$.

Table 2. Expression of nerve growth factor (NGF) integrated optical density value.

\begin{tabular}{lcc}
\hline Group & Cases $(\mathrm{N})$ & NGF integrated optical density value \\
\hline Patients & 48 & $3867.52 \pm 1260.36$ \\
controls & 34 & $698.98 \pm 264.32$ \\
t-value & 1.376 & 3.967 \\
P value & 0.062 & $0.005 *$ \\
\hline
\end{tabular}

*Compared with the healthy control group, $\mathrm{P}<0.01$.

\section{DISCUSSION}

Cerebral aneurysms in the cerebral artery are accompanied by high pressure on the partial wall cavity, which causes thinning of the cerebral artery wall and abnormal bulging. The major causes include congenital malformations, infection, atherosclerosis, hypertension, trauma, and familial predisposition. In the branch and trunk of the cerebral artery bifurcation, the muscular congenital defect of the arterial wall is hardest hit by long-term blood pressure and physical impact, so these points will project outwardly and gradually form an aneurysm. In this study, the tumor site was identified with CTA. Since the site of the occurrence of cerebral aneurysms has some unique characteristics, the tumor may rupture at any time. Nervousness, 
agitation, excessive fatigue, and strenuous exercise can cause a blood pressure increase, which can easily lead to bleeding. Furthermore, this disease can threaten the patient's life.

Currently, the clinical methods for treating cerebral aneurysms are mainly medical and surgical. Medical treatment involves controlling the risk factors of ruptured tumors to relieve the stimulation of the tumor wall. Additional methods are based on the use of medical devices to treat tumors, including surgical occlusion of the aneurysm neck, aneurysm neck ligation, isolation of the aneurysm, and wrapping of the aneurysm. To date, the mechanisms underlying the occurrence and development of aneurysms are still not very clear. While many studies have shown that patients with cerebral aneurysms exhibit gene expression changes in the body that are related to $V E G F, T G F-\beta 1, S P-1$, and $M M P$ (Hughes et al., 2005), at this time, no firm conclusions can be formed. Therefore, the mechanisms underlying the development of cerebral aneurysms require further research and exploration.

NGF, one of the first identified neurotrophic factors, functions by combining with a specific nerve growth factor receptor on target cells. Research shows that it is expressed in various circulatory and immune systems (Chang et al., 2011). There are two main types of coupled receptors of NGF: TrkA and p75. The former is a high-affinity receptor, while the latter is a low-affinity receptor. One study showed that in cell lines that highly express TrkA and p75, such as those of pancreatic cancer, ovarian cancer, liver cancer, etc., elevated expression of NGF can stimulate the proliferation of cells and increase their invasion ability. If a blocker of TrkA is added, no cell proliferation or invasion is observed (Wojtkowiak et al., 2011). In addition, some research indicates that NGF combined with $p 75$ will greatly decrease the growth and invasion ability of cells. These data indicate that the invasion of cells induced by NGF may be caused by its combination with TrkA (Hoare et al., 2011). Based on previous studies, our research aimed to detect the expression level of NGF in cerebral aneurysm tissue to find a relationship between them. This article provides theoretical support for further study of the mechanism of cerebral aneurysms. The results of this research show that the expression of NGF can increase cerebral aneurysm risk and result in a poorer prognosis of this disease. However, the higher the expression of $\mathrm{p} 75$, the less-frequently cell proliferation and invasion are observed. At the same time, a better prognosis will benefit this disease. Therefore, evaluation of the expression of two receptors in cerebral aneurysm tissue is very important for prognosis. Further experiments are needed to validate the expression of these two receptors in cerebral aneurysms.

CTA is a noninvasive angiography technique that uses a superficial surface intravenous contrast agent to reach the target vessel through the blood circulation. Computed tomography scanning was used to collect information and to maximize the visibility of the aneurysm's morphology and size, the angular relationship of the tumor with the aneurysm, the main source of the feeding artery, the tumor thrombus, and vessel wall calcification. This method also shows the relationship between the aneurysm anatomy and organ tissue, and it can improve the therapeutic effect (Bock et al., 2010). In this study, the CTA technique was used to detect the brain aneurysms, and the tumor resection was clearly visible. This research lays the foundation for further surgery and pathological data collection.

In summary, this study sought to detect changes in the gene expression of cerebral aneurysms. The increase in NGF expression levels observed in this study is in agreement with that observed in previous studies. However, the over-expression of NGF and whether it leads to increased tumor cell proliferation and invasion capacity will require further study of the corresponding receptor on the cell surface of the tumor location. 


\title{
Conflicts of interest
}

The authors declare no conflict of interest.

\section{ACKNOWLEDGMENTS}

\author{
Research supported by the Shandong Project for Technology and Science \\ (\#2011YD21004).
}

\section{REFERENCES}

Bock N, Riminucci A, Dionigi C, Russo A, et al. (2010). A novel route in bone tissue engineering: magnetic biomimetic scaffolds. Acta Biomater. 6: 786-796.

Chan A, Orme RP, Fricker RA and Roach P (2013). Remote and local control of stimuli responsive materials for therapeutic applications. Adv. Drug Deliv. Rev. 65: 497-514.

Chang B, Sha X, Guo J, Jiao Y, et al. (2011). Thermo and pH dual responsive, polymer shell coated, magnetic mesoporous silica nanoparticles for controlled drug release. J. Mater. Chem. 21: 9239-9247.

Golledge J, Clancy P, Moran C, Biros E, et al. (2010). The novel association of the chemokine CCL22 with abdominal aortic aneurysm. Am. J. Pathol. 176: 2098-2106.

Hoare T, Timko BP, Santamaria J, Goya GF, et al. (2011). Magnetically triggered nanocomposite membranes: a versatile platform for triggered drug release. Nano Lett. 11: 1395-1400.

Hughes S, El Haj AJ and Dobson J (2005). Magnetic micro- and nanoparticle mediated activation of mechanosensitive ion channels. Med. Eng. Phys. 27: 754-762.

Martinez-Pinna R, Barbas C, Blanco-Colio LM, Tunon J, et al. (2010). Proteomic and metabolomic profiles in atherothrombotic vascular disease. Curr. Atheroscler. Rep. 12: 202-208.

Michel JB, Martin-Ventura JL, Egido J, Sakalihasan N, et al. (2011). Novel aspects of the pathogenesis of aneurysms of the abdominal aorta in humans. Cardiovasc. Res. 90: 18-27.

O’Hare AM, Fanning NF, Ti JP, Dunne R, et al. (2010). HydroCoils, occlusion rates, and outcomes: a large single-center study. AJNR Am. J. Neuroradiol. 31: 1917-1922.

Ramos-Mozo P, Rodriguez C, Pastor-Vargas C, Blanco-Colio LM, et al. (2012). Plasma profiling by a protein array approach identifies IGFBP-1 as a novel biomarker of abdominal aortic aneurysm. Atherosclerosis 221: 544-550.

Schwenk JM, Igel U, Kato BS, Nicholson G, et al. (2010). Comparative protein profiling of serum and plasma using an antibody suspension bead array approach. Proteomics 10: 532-540.

Seidel M, Herguijuela M, Forkert R and Otten U (2010). Nerve growth factor in rheumatic diseases. Semin. Arthritis Rheum. 40: 109-126.

van Kuijk JP, Flu WJ, Chonchol M, Bax JJ, et al. (2010). Metabolic syndrome is an independent predictor of cardiovascular events in high-risk patients with occlusive and aneurysmatic peripheral arterial disease. Atherosclerosis 210: 596-601.

Wojtkowiak JW, Verduzco D, Schramm KJ and Gillies RJ (2011). Drug resistance and cellular adaptation to tumor acidic pH microenvironment. Mol. Pharm. 8: 2032-2038. 